\title{
CHARACTERIZATIONS FOR APPROXIMATELY NORMAL ALGEBRAS
}

\author{
DONALD R. CHALICE ${ }^{1}$
}

1. Introduction. Let ${ }^{-} C(X)$ be the algebra of complex valued continuous functions on a compact Hausdorff space $X$. A function algebra $A$ is a uniformly closed separating subalgebra of $C(X)$ containing the constants.

Definition 1.1. $A$ is approximately normal on $X$ if for any two disjoint closed sets $E, F$ in $X$ and $\epsilon>0$ there is a function $f$ in $A$ such that $|f|<\epsilon$ on $E$ and $|1-f|<\epsilon$ on $F$.

In this note we characterize approximately normal algebras and their essential sets. As applications we obtain a "belonging" result of Wilken [9] and show that the only algebra which restricts to all continuous functions on each of a countable number of closed sets whose union is $X$ is the algebra $C(X)$.

Notation. For a closed set $F \subset X$ and $f \in C(X),\|f\|_{F}$ will denote the sup norm of $f \mid F$, the restriction of $f$ to $F . A_{F}$ will be the uniform closure of the algebra of restrictions, $A \mid F=\{f \mid F: f \in A\} . M(A)$ will denote the maximal ideal space of $A$ and $\hat{A}$ the algebra of Gelfand transforms of $A$. $A$-hull $(F)=\left\{p \in M(A):|\hat{f}(p)| \leqq\|f\|_{F}\right.$ for all $\left.f \in A\right\}$. For $p \in M(A)$, a positive regular Borel measure $\mu_{p}$ on $X$ represents $p$ if $f(p)=\int f d \mu_{p}$ for all $f \in A$. For a bounded regular Borel measure $\mu$ on $X$, supp $\mu$, the closed support of $\mu$, is the complement of the largest open set of $|\mu|$-measure 0 .

2. Characterization of approximate normality. For a closed set $F$ in $X, M\left(A_{F}\right)=A$-hull $(F)$ [6]. The following is immediate.

Lemma 2.1. Let $A$ be a function algebra on $X$. Then for any closed set $F$ in $X, A$-hull $(F)=\left\{p \in M(A): \exists\right.$ a representing measure $\mu_{p} \ni:$ supp $\left.\mu_{p} \subset F\right\}$.

The following generalizes a result Ryff obtained for the disk algebra using Mergelyan's theorem [7].

LEMMA 2.2. Let $\mu$ be either a representing measure or an extreme point of the ball of $A^{\perp}$, the annihilator of the function algebra $A$. Then if $S=\operatorname{supp} \mu, A$-hull $(S)$ is connected.

Received by the editors October 20, 1967.

1 Part of the material here appears in the author's doctoral dissertation at NorthWestern University. The research was supported in part by the National Science Foundation. The author wishes to thank Professor G. M. Leibowitz for his guidance throughout this research. 
Proof. It is well known that in either case $S=\operatorname{supp} \mu$ is a set of antisymmetry for $A_{S}$ [3]. Thus $A_{S}$ is antisymmetric and it follows from Silov's idempotent theorem [5] that $M\left(A_{S}\right)=A$-hull( $\left.S\right)$ must be connected.

THEOREM 2.3. The following are equivalent.

(i) $A$ is approximately normal on $X$.

(ii) Every representing measure for $A$ on $X$ has connected closed support.

(iii) For each pair of disjoint closed sets $E, F$ in $X, A$-hull $(E \cup F)$ $=A$-hull $(E) \cup A$-hull $(F)$ and $A$-hull $(E) \cap A$-hull $(F)=\varnothing$.

(iv) For each closed set $F$ in $X$, the canonical map $G^{\prime} \rightarrow G^{\prime} \cap F$ from the open closed sets of $M\left(A_{F}\right)$ to the open closed sets of $F$ is 1-1 and onto.

(v) For each closed set $F$ in $X$, the canonical map from the components of $F$ to the components of $M\left(A_{F}\right)$ is 1-1 and onto.

If $A$ is approximately normal then the inverse map for (iv) and the map in (v) are both given by taking the $A$-hull.

Proof. (i) $\Rightarrow$ (ii). Let $\mu$ be a representing measure for $A$ concentrated on $X$. If supp $\mu=E \cup F$ where $E$ and $F$ are disjoint closed sets then by approximate normality, the characteristic function of $E$. $\chi_{E}$ is in $A_{E \cup F}$, contradicting the antisymmetry of supp $\mu$.

(ii) $\Rightarrow$ (iii). If $E, F$ are disjoint closed sets in $X$, then by Lemma 2.1 $A$-hull $(E \cup F) \subset A$-hull $(E) \cup A$-hull $(F)$ and so we have equality If $p \in A$-hull $(E) \cap A$-hull $(F)$ then there are representing measures $\mu_{1}, \mu_{2}$ for $p$ concentrated on $E, F$ respectively so that $1 / 2 \mu_{1}+1 / 2 \mu_{2}$ represents $p$ and does not have connected support, contradicting (ii).

(iii) $\Rightarrow$ (i). If $E, F$ are disjoint closed sets in $X$ then $M\left(A_{E \cup F}\right)$ $=A$-hull $(E \cup F)=A$-hull $(E) \cup A$-hull $(F)$ (disjoint). Thus by the Silov idempotent theorem $\chi_{E} \in A_{E \cup F}$ and approximate normality follows.

(ii) $\Rightarrow$ (iv). Let $F$ be a closed set in $X$. There is a 1-1 correspondence between the open closed sets of $M\left(A_{F}\right)$ and the continuous characteristic functions in $\hat{A}_{F}$. Since $F$ is the Silov boundary of $A_{F}$ this gives a 1-1 map from the open closed sets of $M\left(A_{F}\right)$ into the open closed sets of $F$ which is "intersection." By approximate normality any open closed set in $F$ gives rise to a characteristic function in $A_{F}$ so that this map is onto.

If $G^{\prime}$ is open closed in $M\left(A_{F}\right)$ and $\mu_{p}$ represents $p \in G^{\prime}$ then by antisymmetry supp $\mu_{p} \subset G^{\prime}$. Thus $G^{\prime}=A$-hull $\left(G^{\prime} \cap F\right)$ by Lemma 2.1 .

(iv) $\Rightarrow$ (v). We use the fact that the components are intersects of open closed sets. Thus, normality and compactness shows that the map components $(F) \rightarrow$ components $\left(M\left(A_{F}\right)\right)$ is onto. Similarly assuming (iv), two distinct components in $F, C_{1}$ and $C_{2}$ are contained in 
disjoint open closed sets in $F$. The corresponding open closed sets in $M\left(A_{F}\right)$ must be disjoint and hence $C_{1}$ and $C_{2}$ are contained in distinct components in $M\left(A_{F}\right)$, so that the map is 1-1.

(v) $\Rightarrow$ (ii) is clear by Lemma 2.2 .

If $C^{\prime}$ is the component in $M\left(A_{F}\right)$ corresponding to $C$ then $C^{\prime}$ is an intersection of open closed sets and hence as above if, $p \in C^{\prime}$ and $\mu_{p}$ represents $p$, then supp $\mu_{p}$ is contained in $C^{\prime}$. Thus $C^{\prime}=A$-hull $\left(C^{\prime} \cap F\right)$. Note that $C=C^{\prime} \cap F$ since by (iv) $C^{\prime} \cap F$ must be connected.

One notes that (a) $R(X)$ (for a compact set $X$ in the plane) is approximately normal iff $X$ has no interior [9], (b) a pervasive algebra on $X$ is approximately normal iff $X$ is connected, and (c) every Dirichlet algebra is approximately normal. Thus the following result of Coburn [2] originally obtained via Toeplitz operators, follows.

Corollary 2.4. If $A$ is Dirichlet on $X$ then every representing measure for $A$ on $X$ has connected closed support.

CoRollary 2.5. If $A$ is approximately normal then every extreme point of $b\left(A^{\perp}\right)$ has connected closed support.

The above allows the following conceptual proof of a "belonging" result of Wilken [9].

CoRollary 2.6. If $A$ is approximately normal on $X$ and $\left\{C_{\alpha}\right\}$ is the family of components of $X$ and if $f \in C(X)$ has $f\left|C_{\alpha} \in A\right| C_{\alpha}$ for all $\alpha$, then $f \in A$.

Proof. It suffices to show that $f$ is annihilated by each extreme point of the ball of $A^{\perp}$. But each such extreme point is concentrated on a component and thus the result follows from the Kreln-Milman theorem.

REMARK. Steen [8] has constructed an essential approximately normal Swiss cheese algebra $R(X)$ on a connected planar set $X$ which is not antisymmetric, so it follows that components are not necessarily antisymmetric for essential approximately normal algebras. Conversely, Corollary 2.5 shows that approximately normal algebras do contain many connected sets of antisymmetry. Although we have not been able to construct a counterexample, it seems unlikely that all closed sets of antisymmetry will be connected.

3. Characterization of the essential set. The essential set for an algebra $A$ on $X$ is the smallest closed set supporting every bounded regular Borel measure on $X$ annihilating $A$. Alternately it is the smallest closed set $E_{X}$ in $X$ such that $f \in C(X)$ and $f\left|E_{X} \in A\right| E_{X} \Rightarrow f \in A$ $[2]$. 
For $A$ having metrizable ideal space $M=M(A)$, Mullins [4] has characterized the essential set on $M(A)$ by $E_{M}=M \sim P$ where $P=\left\{x \in M: \exists\right.$ a closed nbhd $\bar{V}_{x}$ of $x$ with $\left.A \mid \bar{V}_{x}=C\left(\bar{V}_{x}\right)\right\}$. Such a characterization fails in general for $X \subsetneq M(A)$, as he points out. However it does hold if $A$ is approximately normal on $X$. To do this we use the following "2-local" belonging property of approximately normal algebras [9].

Lemma 3.1. Let $A$ be approximately normal on $X$ and let $f \in C(X)$. If there are open sets $U_{1}, U_{2}$ such that $X=U_{1} \cup U_{2}$ and $f\left|U_{1} \in A\right| U_{1}$ and $f\left|U_{2} \in A\right| U_{2}$ then $f \in A$.

Proposition 3.2. If $A$ is approximately normal on $X$, then $E_{X}$ $=X \sim P$ where $P=\left\{x \in X: \exists\right.$ a closed nbhd $\bar{V}_{x}$ of $x$ with $\left.A \mid \bar{V}_{x}=C\left(\bar{V}_{x}\right)\right\}$.

Proof. Evidently $X \sim P \subset E_{X}$. Now suppose $x \in P$. Then choose open sets $U$ and $W$ such that $x \in U \subset \bar{U} \subset W$ and $A \mid \bar{W}=C(\bar{W})$. Suppose that $f \in C(X)$ and $f=0$ on $X \sim U$. Then $f|X \sim \bar{U} \in A| X \sim \bar{U}$ and $f|W \in A| W$. As $X=W \cup X \sim \bar{U}$, by the lemma $f \in A$. Thus $E_{X} \subset X \sim U$ by the defining property of the essential set.

Using the characterization obtained for $E_{M}$, Mullins goes on to show that $A$ cannot restrict to all continuous functions on each of a countable number of closed sets whose union is $M(A)$ (metrizable). We use (3.2) to show some slightly stronger results.

Definition. Let $p \in M(A)$. A Jensen measure $\mu_{p}$ for $p$ on $X$ is a representing measure for $p$ on $X$ satisfying $\log |f(p)| \leqq \log \int|f| d \mu_{p}$ for all $f \in A$. Bishop has shown that any point $p \in M(A)$ always has a Jensen measure on $X$.

It follows that if $F$ is a closed set in $X$ and $A \mid F$ is closed and if $\mu_{p}$ is a Jensen measure with $\mu_{p}(F)>0$ then $p \in M(A \mid F)$. In particular a Jensen measure always has either perfect closed support or is a pointmass.

Corollary 3.3. If $A$ is a function algebra on $X=\bigcup_{i=1}^{\infty} F_{i}$ where $F_{i}$ are closed and $A \mid F_{i}=C\left(F_{i}\right)$ for each $i$, then $A=C(X)$.

Proof. First $A$ is approximately normal on $X$. For if $p \in M(A)$ and $\mu_{p}$ is a Jensen measure for $p$ then $\mu_{p}\left(F_{i}\right)>0$ for some $i$ and thus $\mu_{p}$ is the point-mass at $p$. Thus for any closed set $F$ in $X, M\left(A_{F}\right)=F$. It follows that $A$ is approximately normal.

To show $A=C(X)$ we show the essential set $E$ of $A$ on $X$ is empty. But $E=\bigcup_{i=1}^{\infty} E \cap F_{i}$ and by the Baire category theorem one set $E \cap F_{i}$ has nonempty interior with respect to $E$. But this means that for some nonempty closed neighborhood $\bar{V}$ in $E, A \mid \bar{V}=C(\bar{V})$ and this contradicts the characterization of $E$ given by (3.2). 
We point out that the last part of the proof is exactly that used by Mullins in his original result. (3.3) has also been obtained by Mullins and by Gamlin and Wilken in a different fashion. As another application of (3.2) we obtain a slightly stronger result.

Corollary 3.4. Let $F$ be a subset of $X$ which is closed and has no perfect subsets. If $X \sim F=\cup_{i=1}^{\infty} F_{i}$ where $F_{i}$ are closed and $A \mid F_{i}=C\left(F_{i}\right)$ for all $i$, then $A=C(X)$.

Proof. If $\mu_{p}$ is any Jensen measure on $X$ then either $\mu_{p}$ is supported in $F$ or $\mu_{p}\left(F_{i}\right)>0$ for some $i$. Since $F$ contains no perfect subsets and since $A \mid F_{i}=C\left(F_{i}\right)$, in either case, $\mu_{p}$ must be the point-mass at $p$. It follows that $M(A)=X$ and that $A$ is approximately normal on $X$.

By (3.3) if $\bar{V}$ is a closed neighborhood in $X \sim F$ then $A \mid \bar{V}=C(\bar{V})$. Thus $E$, the essential set of $A$ on $X$, is in $F$. Since $X=M(A), E$ is perfect. Thus $E$ is empty and $A=C(X)$.

The last proof used some techniques from an elegant proof by $\mathrm{T}$. Read of the result of Rudin (when each $F_{i}=\varnothing$ ).

\section{REFERENCES}

1. H. S. Bear, Complex function algebras, Trans. Amer. Math. Soc. 90 (1959), 383-393.

2. L. A. Coburn, Generalized Toeplitz operators and function algebras, Abstract 619-149, Notices Amer. Math. Soc. 12 (1965), 101.

3. I. Glicksberg, Measures orthogonal to algebras and sets of antisymmetry, Trans. Amer. Math. Soc. 105 (1962), 415-435.

4. R. Mullins, The essential set of function algebras, Proc. Amer. Math. Soc. 18 (1967), 271-274. 1960.

5. C. Rickart, General theory of Banach algebras, Van Nostrand, Princeton, N. J.,

6. H. Rossi, The local maximum modulus theorem, Ann. of Math. 72 (1960), 1-11.

7. J. V. Ryff, "The support of representing measures for the disk algebra," in Function algebras (Proc. Internat. Sympos. on Function Algebras, Tulane Univ., 1965), Scott-Foresman, Chicago, Ill., 1966, pp. 112-117.

8. L. A. Steen, On uniform approximation by rational functions, Proc. Amer. Math. Soc. 17 (1966), 1007-1011.

9. D. R. Wilken, "Approximate normality and function algebras on the interval and the circle," in Function algebras, edited by F. Birtel (Proc. Internat. Sympos. on Function Algebras, Tulane Univ., 1965), Scott-Foresman, Chicago, Ill., 1966, pp. 98-111.

NORTHWESTERN UNIVERSITY AND

Western Washington State College 